hep-th/0405085

UUITP-09/04

HIP-2004-17/TH

CPHT-RR 018.0504

LPTHE-04-08

\title{
Generalized complex manifolds and supersymmetry
}

\author{
Ulf Lindström ${ }^{a b}$, Ruben Minasian $^{c}$, Alessandro Tomasiello $^{c}$ and Maxim Zabzine ${ }^{d e}$ \\ ${ }^{a}$ Department of Theoretical Physics \\ Uppsala University, Box 803, SE-751 08 Uppsala, Sweden \\ ${ }^{b}$ HIP-Helsinki Institute of Physics \\ P.O. Box 64 FIN-00014 University of Helsinki, Suomi-Finland \\ ${ }^{c}$ Centre de Physique Théorique, Ecole Polytechnique \\ 91128 Palaiseau Cedex, France \\ ${ }^{d}$ LPTHE, Université Pierre et Marie Curie, Paris VI \\ 4 Place Jussieu, 75252 Paris Cedex 05, France \\ ${ }^{e}$ Institut Mittag-Leffler, Auravägen 17, S-182 62 Djursholm, Sweden
}

\begin{abstract}
We find a worldsheet realization of generalized complex geometry, a notion introduced recently by Hitchin which interpolates between complex and symplectic manifolds. The two-dimensional model we construct is a supersymmetric relative of the Poisson sigma model used in context of deformation quantization.
\end{abstract}




\section{Introduction}

The recently developed notion of generalized complex geometry naturally extends and unifies complex and symplectic geometries, in general interpolating between the two $[1,2,3]^{1}$. There have been many hints that this geometry should be relevant to string theory. In this paper, we realize this expectation from a world-sheet perspective.

The reasons to believe that generalized complex geometry should fit naturally in string theory basically all stem from the fact that the formalism puts the tangent $T$ and the cotangent bundle $T^{*}$ on the same footing, considering pairs $(v, \xi)$ in $T \oplus T^{*}$. The basic objects of the formalism, generalized (almost) complex structures, are endomorphisms of this bundle, and admit an action not only under diffeomorphisms but also under a twoform. As we will see, this action is essentially a change in the string theory $B$-field. A related remark is that the structure group of this bundle is $\mathrm{SO}(d, d)$, which indicates a relation to the string theory $\mathrm{T}$-duality group. This is strengthened by the interpolation between complex and symplectic geometry, which are mirrors in string theory.

The formalism has in fact already found an application recently, from a perspective different from the one in this paper. A mirror symmetry transformation was proposed in [5] for manifolds of SU(3) structure, generalizing the case of Calabi-Yau manifolds with NS flux, considered in $[6,7]$. As it turns out, mirror symmetry can be expressed naturally in terms of the $T \oplus T^{*}$ formalism.

In [5] mirror symmetry is expressed as an exchange of two pure spinors. These objects appear in many disparate contexts, depending on which Clifford algebra one is considering. In case of Clifford $(9,1)$ they play a role in Berkovits superstring [8], and in the case of Clifford $(d)$ they can be used to define twistor spaces for manifolds of dimension $d[9]$. In the present paper the relevant spinors are those of the Clifford algebra naturally built on $T \oplus T^{*}$, which is Clifford $(d, d)$. The same way as usual Clifford $(d)$ spinors can be realized in terms of $(0, p)$ forms on an almost complex manifold, Clifford $(d, d)$ spinors can be realized as formal sums of forms of mixed degrees. Pure spinors are then those which have a stabilizer of maximal dimension, which can be translated into an algebraic condition that we will review later. On a $\mathrm{SU}(d)$ structure manifold we can give two prototypical examples, which are also those exchanged by mirror symmetry: the $(d / 2,0)$ form $\Omega$ and an exponential of the two-form, $e^{i J}$.

These pure spinors play a role in the $T \oplus T^{*}$ formalism: there is a correspondence between generalized complex structures and pure spinor lines. These two complementary pictures of

\footnotetext{
${ }^{1}$ In fact before the Hitchin's work [1] the algebraic aspects of a generalized complex (Kähler) geometry has been discussed in the physics literature [4].
} 
the formalism can be seen as the two complementary pictures of string theory - from the world-sheet and from the supergravity point of view. Pure spinors naturally emerge in the low-energy context, in which, in particular, the above mentioned mirror symmetry proposal was formulated. In this paper, we are going to see how generalized complex structures emerge from the world-sheet point of view.

Another aspect which is taken into account naturally by the formalism is the following. Mirror symmetry was defined in [5] in the class of manifolds of SU(3) structures. This was however a simplification. Mirror symmetry as defined in [5] is inspired by T-duality along three directions. In certain cases, essentially when the $B$-field has more than one leg along the T-dualized directions, ${ }^{2}$ the result of T-duality only makes sense as a "non-geometric" background. Usually, one thinks geometric quantities are sections of bundles associated with the frame bundle: they transform from chart to chart under diffeomorphisms. In string theory, the symmetry group is larger than diffeomorphisms. One can indeed use the $\mathrm{SO}(d, d)$ invariance mentioned above. Then, there may exist more general $\mathrm{SO}(d, d)$-valued transition functions, apart from the the usual Diff-valued ones. This will for example mix metric and $B$-field, making them not well-defined separately. In this situation one speaks of a nongeometrical background. This possibility has been emphasized in many papers; ScherkSchwarz compactifications are for example of this type, and also the ones in $[10,11] .{ }^{3}$ Let us also emphasize that we are not assuming the existence of global isometries, and not doing T-duality. $\mathrm{SO}(d, d)$ only appears as a structure group.

Hopefully, the structure described above will allow the formulation of mirror symmetry using pure spinors to be extended to "non-geometrical" situations.

The present paper realizes $\mathrm{SO}(d, d)$ covariance and describes generalized complex geometry. The idea is simple and is introduced in a paper by one of the present authors [12]. The usual sigma model only contains fields in $T$, the images under the differential of the map $X$ from the world-sheet to the target space. It does not contain objects in $T \oplus T^{*}$. A related model is the Poisson sigma model which does contain fields both in $T$ and $T^{*}$, and was used in the context of deformation quantization [13]. We mimic the structure of the Poisson sigma model for the usual one. We double the number of degrees of freedom introducing new fields $\eta$ valued in the tangent $T^{*}$, and write an action for these $2 d$ fields classically equivalent to the usual sigma model.

A difference between the two actions (the first-order one and the usual second-order sigma-model) is that while a second-order action is fully determined by the metric and a

\footnotetext{
${ }^{2}$ For simplicity, this case was not considered in [5].

${ }^{3}$ Our interest in these matters owes much to a conversation with S. Hellerman, who also made the above remark about non-geometrical mirror symmetry.
} 
closed 3-form $H$, a first order action needs a section $E$ of the $O(d, d)$ bundle $T \oplus T^{*}$. Any two such first-order actions are equivalent, i.e., lead to the same set of equations of motion, as long as they are transformed into each other by an action of a closed 2-form $b$. This puts the $b$-transform on equal footing with diffeomorphisms. This already captures some features of the formalism of generalized complex structures.

More differences between the first and second order forms show up when we try to supersymmetrize the action. We are used to the idea that requiring the action to be supersymmetric constrains the target space geometry. In the second-order action this does not involve a generalized complex structure, only complex structures. In this paper we analyze the conditions under which the first order action has additional supersymmetries. This was done for $N=(2,2)$ supersymmetry in [12] going partly on-shell and is done here completely off-shell for $N=(2,0)$. Given what we already mentioned, it is fair to expect the appearance of generalized complex structures.

In this paper we study models with $N=(2,0)$ supersymmetry (in the absence of boundaries), and find a generalized complex geometry. We consider three different cases, and the realization of the generalized complex geometry depends on the details. In particular, for a special case, at algebraic level we recover the $N=(2,2)$ geometry discovered in $[14]^{4}$. The first order action serves as a basis for T-duality. Since T-duality mixes the right and left sectors [16], this form of the action probes all models related by such transformations.

There are many directions in which the present work might be extended. An obvious one is the inclusion of boundaries. In particular this may clarify the cases discussed in $[17,18]$ for which a geometrical interpretation is lacking. Further, in topological models, the relevance of generalized complex structures has been demonstrated in [19] (see also [20]). It would be interesting to twist the physical model discussed in this paper to reproduce those results in a more general setting.

The structure of the paper is as follows. In sec. 2 we introduce the first-order action and some notation. A brief review of generalized complex geometry is given in sec. 3 . We phrase the integrability conditions in local coordinates. Sec. 4 contains a discussion of the topological model. It represents the most general geometric situation. As the $T \oplus T^{*}$ formalism allows for twisting with 3 -form $H$ it is natural to examine the twisted construction in our context as well. This is done in sec. 5 where we discuss the WZ-term. The $(2,0)$ sigma model is presented in section 6 and the geometry of the target space is discussed. Finally, we gather the most technical part of our computations, namely the closure of the supersymmetry algebra, in an appendix.

\footnotetext{
${ }^{4}$ See also [15], for recent developments.
} 


\section{First order actions}

In this Section we describe the class of two dimensional models which are relevant for our discussion.

We start by introducing the standard bosonic sigma model. This model has a single bosonic real field, $X . X$ is a map from a two-dimensional world-sheet $\Sigma$ (without a boundary) to a manifold $\mathcal{M}$ equipped with a metric $g_{\mu \nu}$ and a closed three form $H_{\mu \nu \rho}$. The action of the model is

$$
S=\frac{1}{2} \int g_{\mu \nu}(X) d X^{\mu} \wedge * d X^{\nu}+B_{\mu \nu}(X) d X^{\mu} \wedge d X^{\nu}
$$

where $H=d B$ on some patch. Although $B$ is used to write the action (2.1) down the theory depends only on the three form $H$.

We introduce a globally defined two-form $b_{\mu \nu}$ on $\mathcal{M}$. Then we can define the following tensors

$$
E_{\mu \nu}=g_{\mu \nu}+b_{\mu \nu}, \quad E^{\mu \lambda} E_{\lambda \nu}=\delta_{\nu}^{\mu}, \quad G^{\mu \nu}=\frac{1}{2}\left(E^{\mu \nu}+E^{\nu \mu}\right), \quad \theta^{\mu \nu}=\frac{1}{2}\left(E^{\mu \nu}-E^{\nu \mu}\right)
$$

If $g$ is a Riemannian metric then $G$ is also a Riemannian metric. We can introduce a new field $\eta$ which is a differential form on $\Sigma$ taking values in the pull-back by $X$ of the cotangent bundle of $\mathcal{M}$, i.e. a section of $X^{*}\left(T^{*} \mathcal{M}\right) \otimes T^{*} \Sigma$. There exists a first order action [21, 12]

$$
S=\int \eta_{\mu} \wedge d X^{\mu}+\frac{1}{2} \theta^{\mu \nu} \eta_{\mu} \wedge \eta_{\nu}+\frac{1}{2} G^{\mu \nu} \eta_{\mu} \wedge * \eta_{\nu}+\frac{1}{2}(B-b)_{\mu \nu} d X^{\mu} \wedge d X^{\nu}
$$

which is equivalent to (2.1) upon the integration of $\eta$. Following the terminology proposed in [22] we call $(g, b)$ the closed string data and $(G, \theta)$ the open string data. We would like to stress one evident, but nevertheless important point: Despite the fact that the actions (2.1) and (2.3) are classically equivalent we need slightly different geometrical data to define them. For the second order action $(2.1)$ we need $(\mathcal{M}, g, H)$ while for the first order action (2.3) $(\mathcal{M}, g, b, H)$. If $d b=0$ then all first order actions with different $b$ are equivalent to the same second order action. Hence two first order actions with $E^{\mu \nu}$ and $\tilde{E}^{\mu \nu}$ are physically equivalent if either $E$ and $\tilde{E}$ are related by diffeomorphism or by a shift of the closed form (b-transform), namely $\left(E_{\mu \nu}-\tilde{E}_{\mu \nu}\right) \in \Omega_{\text {closed }}^{2}(\mathcal{M})$. The symmetry group relating the different (but physically equivalent) first order actions is the semidirect product of $\operatorname{Diff}(\mathcal{M})$ and $\Omega_{\text {closed }}^{2}(\mathcal{M})$. This observation will play an important role in further discussion ${ }^{5}$.

\footnotetext{
${ }^{5}$ In this context we have a comment which is not directly relevant to the subject of this paper. Considering the properties of the first order action we could define string theory in the following fashion: Choose an open cover $\left\{U_{\alpha}\right\}$ of a manifold $\mathcal{M}$. For each chart $U_{\alpha}$ define the first order action $S_{\alpha}$ using $E_{\alpha}$ and on the intersection $U_{\alpha} \cap U_{\beta}$ glue the $E$ 's using the semidirect product of $\operatorname{Diff}(\mathcal{M})$ and $\Omega_{\text {closed }}^{2}(\mathcal{M})$. Now $\left(G_{\alpha}, \theta_{\alpha}\right)$ are not tensors in the usual sense anymore since we glue them on $U_{\alpha} \cap U_{\beta}$ using not only $\operatorname{Diff}(\mathcal{M})$. However this "exotic" prescription does not change the physics. This remark is related to the discussion of non-geometrical string theories in $[10,11]$. We hope to discuss these issues in detail elsewhere.
} 
Another interesting property is that the action (2.3) includes the known two-dimensional topological field theories as degenerate limits. Namely if $G=0$ and $d(B-b)=0$ then the action (2.3) corresponds to the Poisson sigma model introduced in [23, 24], provided that $\theta$ is a Poisson tensor. In the case $G=0$ and $d(B-b) \neq 0$ the model can be related to more general type of topological theory, the WZ-Poisson sigma model [25], assuming some specific differential condition between $\theta$ and $d(B-b)$. Presumably these topological models may arise as a result of a decoupling limit in string theory. Although the Poisson and WZPoisson sigma models are not the main subject of this paper many results we present will be applicable to these models as well.

The main goal of this paper is to study the extended supersymmetry of the first order action (2.3). For technical reasons related to supersymmetry it is convenient to switch to light-cone coordinates. Using $(1,0)$ superfields the $N=(1,0)$ supersymmetric version of $(2.3)$ is

$$
S=i \int d^{2} \sigma d \theta\left(D_{+} \Phi^{\mu} S_{=\mu}-S_{+\mu} \partial_{=} \Phi^{\mu}-S_{+\mu} S_{=\nu} E^{\mu \nu}+D_{+} \Phi^{\mu} \partial_{=} \Phi^{\nu}(B-b)_{\mu \nu}\right)
$$

Throughout the paper we use $(+,=)$ as worldsheet indices and $(+,-)$ as two-dimensional spinor indices. We use $(1,0)$ superspace where with a spinor coordinate $\theta$. The covariant derivative $D_{+}$and supersymmetry generator $Q_{+}$satisfy

$$
D_{+}^{2}=i \partial_{+}, \quad Q_{+}=i D_{+}+2 \theta \partial_{+}
$$

where $\partial_{\#}=\partial_{0} \pm \partial_{1}$. In terms of the covariant derivatives, a supersymmetry transformation of a superfield $\Phi$ is given by

$$
\delta_{m} \Phi=i \epsilon_{m}^{+} Q_{+} \Phi, \quad \delta_{m} S_{+}=i \epsilon_{m}^{+} Q_{+} S_{+}, \quad \delta_{m} S_{=}=i \epsilon_{m}^{+} Q_{+} S_{=} .
$$

In terms of $(1,1)$ superfields, the $N=(1,1)$ first order action is given by

$$
S=\int d^{2} \sigma d^{2} \theta\left(D_{+} \Phi^{\mu} S_{-\mu}-S_{+\mu} D_{-} \Phi^{\mu}-S_{+\mu} S_{-\nu} E^{\mu \nu}+D_{+} \Phi^{\mu} D_{-} \Phi^{\nu}(B-b)_{\mu \nu}\right),
$$

where we use the standard notation (see Appendix A in [17]). In what follows we focus on $N=(1,0)$ models. We would like to understand under which assumptions $N=(1,0)$ models admit $N=(2,0)$. However our results may be straightforwardly generalized to the extension of $N=(1,1)$ to $N=(2,1)$ susy.

\section{Generalized complex geometry}

In this Section we review some basic notions and fix notations. Namely we collect general facts concerning the generalized complex structure, see [1] and [2] for further details. Also 
we work out the coordinate form of the integrability conditions for the generalized complex structure.

Let us start by recalling the definition of the standard complex structure on a manifold $\mathcal{M}(\operatorname{dim} \mathcal{M}=d)$. An almost complex structure is defined as a linear map on the tangent bundle $J: T \rightarrow T$ such that $J^{2}=-1_{d}$. This allows the definition of projectors on $T$,

$$
\pi_{ \pm}=\frac{1}{2}\left(1_{d} \pm i J\right)
$$

An almost complex structure is called integrable if the projectors $\pi_{ \pm}$define integrable distributions on $T$, namely if

$$
\pi_{\mp}\left[\pi_{ \pm} X, \pi_{ \pm} Y\right]=0
$$

for any $X, Y \in T$ where [, ] is a standard Lie bracket on $T$.

A generalization of the notion of complex structure has been proposed by Hitchin [1]. In Hitchin's construction $T$ is replaced by $T \oplus T^{*}$ and the Lie bracket is replaced by the appropriate bracket on $T \oplus T^{*}$, the so called Courant bracket. Thus a generalized complex structure is an almost complex structure $\mathcal{J}$ on $T \oplus T^{*}$ whose $+i$-eigenbundle is Courant involutive. This definition is the complex analog of a Dirac structure, a concept unifying Poisson and symplectic geometry, introduced by Courant and Weinstein [26, 27]. A detailed study of generalized complex geometry can be found in Gualtieri's thesis [2].

Now let us give detailed definitions. On $T \oplus T^{*}$ there is a natural indefinite metric defined by $(X+\xi, X+\xi)=i_{X} \xi$. In the coordinate basis $\left(\partial_{\mu}, d x^{\mu}\right)$ we can write this metric as follows

$$
\mathcal{I}=\left(\begin{array}{ll}
0 & 1_{d} \\
1_{d} & 0
\end{array}\right)
$$

A generalized almost complex structure is a map $\mathcal{J}: T \oplus T^{*} \rightarrow T \oplus T^{*}$ such that $\mathcal{J}^{2}=-1_{2 d}$ and that $\mathcal{I}$ is hermitian with respect to $\mathcal{J}, \mathcal{J}^{t} \mathcal{I} \mathcal{J}=\mathcal{I}$. On $T \oplus T^{*}$ there is a Courant bracket which is defined as follows

$$
[X+\xi, Y+\eta]_{c}=[X, Y]+\mathcal{L}_{X} \eta-\mathcal{L}_{Y} \xi-\frac{1}{2} d\left(i_{X} \eta-i_{Y} \xi\right)
$$

This bracket is skew-symmetric but in general does not satisfy the Jacobi identity. However if there is a subbundle $L \subset T \oplus T^{*}$ which is involutive (closed under the Courant bracket) and isotropic with respect to $\mathcal{I}$ then the Courant bracket on the sections of $L$ does satisfy the Jacobi identity. This is a reason for imposing hermiticity of $\mathcal{I}$ with respect to $\mathcal{J}$. One important feature of the Courant bracket is that, unlike the Lie bracket, this bracket has a nontrivial automorphism defined by a closed two-form $b$,

$$
e^{b}(X+\xi)=X+\xi+i_{X} b
$$


such that

$$
\left[e^{b}(X+\xi), e^{b}(Y+\eta)\right]_{c}=e^{b}[X+\xi, Y+\eta]_{c}
$$

We can construct the projectors on $T \oplus T^{*}$

$$
\Pi_{ \pm}=\frac{1}{2}(I \pm i \mathcal{J})
$$

the almost generalized complex structure $\mathcal{J}$ is integrable if

$$
\Pi_{\mp}\left[\Pi_{ \pm}(X+\xi), \Pi_{ \pm}(Y+\eta)\right]_{c}=0
$$

for any $(X+\xi),(Y+\eta) \in T \oplus T^{*}$. This is equivalent to the single statement

$$
[X+\xi, Y+\eta]_{c}-[\mathcal{J}(X+\xi), \mathcal{J}(Y+\eta)]_{c}+\mathcal{J}[\mathcal{J}(X+\xi), Y+\eta]_{c}+\mathcal{J}[X+\xi, \mathcal{J}(Y+\eta)]_{c}=0
$$

which resembles the definition of the Nijenhuis tensor.

To relate to the construction to the physical models we have to reexpress the above definitions in coordinate form. The map $\mathcal{J}$ can be written in the form

$$
\mathcal{J}=\left(\begin{array}{ll}
J & P \\
L & K
\end{array}\right),
$$

where $J: T \mathcal{M} \rightarrow T \mathcal{M}, P: T^{*} \mathcal{M} \rightarrow T \mathcal{M}, L: T \mathcal{M} \rightarrow T^{*} \mathcal{M}$ and $K: T^{*} \mathcal{M} \rightarrow T^{*} \mathcal{M}$ and hence they correspond to the tensor fields, $J_{\nu}^{\mu}, L_{\mu \nu}, P^{\mu \nu}$ and $K_{\mu}{ }^{\nu}$. Then the condition $\mathcal{J}^{2}=-1_{2 d}$ becomes

$$
\begin{aligned}
& J_{\nu}^{\mu}{ }_{\nu}{ }_{\lambda}{ }_{\lambda}+P^{\mu \nu} L_{\nu \lambda}=-\delta_{\lambda}^{\mu}, \\
& J^{\mu}{ }_{\nu} P^{\nu \lambda}+P^{\mu \nu} K_{\nu}{ }^{\lambda}=0, \\
& K_{\mu}{ }^{\nu} K_{\nu}{ }^{\lambda}+L_{\mu \nu} P^{\nu \lambda}=-\delta^{\mu}{ }_{\lambda}, \\
& K_{\mu}{ }^{\nu} L_{\nu \lambda}+L_{\mu \nu} J_{\lambda}^{\nu}=0 .
\end{aligned}
$$

The hermiticity of $\mathcal{I}$ with respect to $\mathcal{J}$ translates into the following conditions

$$
J_{\nu}^{\mu}+K_{\mu}^{\nu}=0, \quad P^{\mu \nu}=-P^{\nu \mu}, \quad L_{\mu \nu}=-L_{\nu \mu}
$$

In local coordinates the integrability condition (3.9) is equivalent to the following four conditions

$$
\begin{aligned}
& J_{[\lambda}^{\nu} J_{\rho], \nu}^{\mu}+J_{\nu}^{\mu} J_{[\lambda, \rho]}^{\nu}+P^{\mu \nu} L_{[\lambda \rho, \nu]}=0 \\
& P^{[\mu \mid \nu} P^{\mid \lambda \rho]}=0 \\
& J_{\nu, \rho}^{\mu} P^{\rho \lambda}+P_{, \nu}^{\rho \lambda} J_{\rho}^{\mu}-J_{\rho, \nu}^{\lambda} P^{\mu \rho}+J_{\nu, \rho}^{\lambda} P^{\mu \rho}-P_{, \rho}^{\mu \lambda} J_{\nu}^{\rho}=0 \\
& J_{\nu}^{\lambda} L_{[\lambda \rho, \gamma]}+L_{\nu \lambda} J_{[\gamma, \rho]}^{\lambda}+J_{\rho}^{\lambda} L_{\gamma \nu, \lambda}+J_{\gamma}^{\lambda} L_{\nu \rho, \lambda}+L_{\lambda \rho} J_{\gamma, \nu}^{\lambda}+J_{\rho}^{\lambda} L_{\lambda \gamma, \nu}=0
\end{aligned}
$$


To summarize, the generalized complex structure $\mathcal{J}$ is defined by three tensor fields $J^{\mu}{ }_{\nu}, L_{\mu \nu}$ and $P^{\mu \nu}$ which satisfy the algebraic conditions (3.11)-(3.15) and the differential conditions (3.16)-(3.19).

The usual complex structure $J$ is embedded in the notion of generalized complex structure

$$
\mathcal{J}=\left(\begin{array}{cc}
J & 0 \\
0 & -J^{t}
\end{array}\right) .
$$

One can check that all properties (3.11)-(3.19) are satisfied provided that $J$ is a complex structure. Also, a symplectic structure is an example of a generalized complex structure

$$
\mathcal{J}=\left(\begin{array}{cc}
0 & -\omega^{-1} \\
\omega & 0
\end{array}\right)
$$

where $\omega$ is an ordinary symplectic structure $(d \omega=0)$. More exotic examples exist and are given by manifolds, that do not admit any known complex or symplectic structure, but do admit a generalized complex structure $[2,28]$.

Consider a generalized complex structure $\mathcal{J}$; a new generalized complex structure can be generated by

$$
\mathcal{J}_{b}=\left(\begin{array}{ll}
1 & 0 \\
b & 1
\end{array}\right) \mathcal{J}\left(\begin{array}{rr}
1 & 0 \\
-b & 1
\end{array}\right)
$$

if $b \in \Omega_{\text {closed }}^{2}(\mathcal{M})$. The structure $\mathcal{J}_{b}$ is integrable due to the fact that the transformation (3.5) is an automorphism of the Courant bracket. The transformation (3.22) is called a $b$-transform and later we will see that this is related to the $b$-transform for the first order actions discussed in the previous Section.

The key feature of a complex manifold is that is locally equivalent to $C^{k}$ via a diffeomorphism. For symplectic manifolds the Darboux theorem states that a symplectic structure is locally equivalent, via diffeomorphism, to the standard symplectic structure $\left(R^{2 k}, \omega\right)$, where

$$
\omega=d x_{1} \wedge d x_{2}+\ldots+d x_{2 k-1} \wedge d x_{2 k} .
$$

For generalized complex manifolds there exists a generalized Darboux theorem [2], which states that in a neighborhood of a regular point ${ }^{6}$ a generalized complex structure on a manifold $\mathcal{M}$ is locally equivalent via a diffeomorphism and a $b$-transform (see (3.22)), to the product of an open set in $C^{k}$ and an open set in the standard symplectic space $\left(R^{d-2 k}, \omega\right)$.

The Courant bracket on $T \oplus T^{*}$ can be twisted by a closed three form $H$. Namely given a closed three form $H$ one can define another bracket on $T \oplus T^{*}$ by

$$
[X+\xi, Y+\eta]_{H}=[X+\xi, Y+\eta]_{c}+i_{X} i_{Y} H .
$$

\footnotetext{
${ }^{6} P$ is a Poisson structure and it will define a symplectic foliation. The point is called regular if $P$ has constant rank in a neighborhood.
} 
This bracket has similar properties to the Courant bracket. Again if a subbundle $L \subset T \oplus T^{*}$ is closed under the twisted Courant bracket and isotropic with respect to $\mathcal{I}$, then the Courant bracket on the sections of $L$ does satisfy the Jacobi identity. Thus in the integrability condition (3.9) the Courant bracket $[,]_{c}$ can be replaced by the new twisted Courant bracket $[,]_{H}$. In local coordinates the new integrability condition is equivalent to four expressions

$$
\begin{aligned}
& J_{[\lambda}^{\nu} J_{\rho], \nu}^{\mu}+J_{\nu}^{\mu} J_{[\lambda, \rho]}^{\nu}+P^{\mu \nu}\left(L_{[\lambda \rho, \nu]}+J_{[\lambda}^{\sigma} H_{\rho] \sigma \nu}\right)=0 \\
& P^{[\mu \mid \nu} P^{\mid \lambda \rho]}=0 \\
& J_{\nu, \rho}^{\mu} P_{\nu}^{\rho \lambda}+P_{, \nu}^{\rho \lambda} J_{\rho}^{\mu}-J_{\rho, \nu}^{\lambda} P^{\mu \rho}+J_{\nu, \rho}^{\lambda} P^{\mu \rho}-P_{, \rho}^{\mu \lambda} J_{\nu}^{\rho}-P^{\lambda \sigma} P^{\mu \rho} H_{\sigma \rho \nu}=0 \\
& J_{\nu}^{\lambda} L_{[\lambda \rho, \gamma]}+L_{\nu \lambda} J_{[\gamma, \rho]}^{\lambda}+J_{\rho}^{\lambda} L_{\gamma \nu, \lambda}+J_{\gamma}^{\lambda} L_{\nu \rho, \lambda}+L_{\lambda \rho} J_{\gamma, \nu}^{\lambda}+J_{\rho}^{\lambda} L_{\lambda \gamma, \nu}+ \\
& +H_{\rho \gamma \nu}-J_{[\rho}^{\lambda} J_{\gamma}^{\sigma} H_{\nu] \lambda \sigma}=0
\end{aligned}
$$

\section{Topological model}

In this Section we consider a toy topological model which will provide a "physical" derivation of generalized complex geometry. Also it will lead to results which will be relevant for the physical model (6.2). The model has the following action

$$
S_{\text {top }}=\int d^{2} \sigma d \theta S_{+\mu} \partial_{=} \Phi^{\mu}
$$

which is part of the action (6.2). This is a topological system which describes the holomorphic maps $\Phi: \Sigma \rightarrow \mathcal{M}$. The model is manifestly $N=(1,0)$ supersymmetric and can be defined over any differential manifold $\mathcal{M}$. We would like to find the restrictions on $\mathcal{M}$ arising from the requirement that the model admits $(2,0)$ supersymmetry.

We have to look for additional (non manifest) supersymmetry transformations. The general transformations of $S_{+}$and $\Phi$ are given by the following expressions

$$
\begin{gathered}
\delta(\epsilon) \Phi^{\mu}=\epsilon^{+} D_{+} \Phi^{\nu} J_{\nu}^{\mu}-\epsilon^{+} S_{+\nu} P^{\mu \nu} \\
\delta(\epsilon) S_{+\mu}=i \epsilon^{+} \partial_{+} \Phi^{\nu} L_{\mu \nu}-\epsilon^{+} D_{+} S_{+\nu} K_{\mu}{ }^{\nu}+\epsilon^{+} S_{+\nu} S_{+\rho} N_{\mu}{ }^{\nu \rho}+ \\
+\epsilon^{+} D_{+} \Phi^{\nu} D_{+} \Phi^{\rho} M_{\mu \nu \rho}+\epsilon^{+} D_{+} \Phi^{\rho} S_{+\nu} Q_{\mu \rho}{ }^{\nu}
\end{gathered}
$$

Classically the Ansatz (4.2) and (4.3) is unique on dimensional grounds and by Lorentz covariance [12]. This Ansatz involves seven different tensors on $\mathcal{M}$. We have to require the standard $N=(2,0)$ supersymmetry algebra, i.e. the manifest and non-manifest supersymmetry transformations commute and the nonmanifest supersymmetry transformations satisfy the following conditions

$$
\left[\delta\left(\epsilon_{2}\right), \delta\left(\epsilon_{1}\right)\right] \Phi^{\mu}=2 i \epsilon_{1}^{+} \epsilon_{2}^{+} \partial_{+} \Phi^{\mu}, \quad\left[\delta\left(\epsilon_{2}\right), \delta\left(\epsilon_{1}\right)\right] S_{+\mu}=2 i \epsilon_{1}^{+} \epsilon_{2}^{+} \partial_{+} S_{+\mu} .
$$


Since the nonmanifest transformations are written in $(1,0)$ superfield then the first requirement is automatically satisfied. Next we have to calculate the commutator of two nonmanifest supersymmetry transformations. The result of the calculation is given in Appendix. Imposing the condition (4.4) implies four algebraic and eleven differential conditions on the seven tensors introduced in (4.2) and (4.3). This fact alone shows how the problem of extended supersymmetry becomes involved when extra fields are introduced.

Before analyzing the algebra in detail it is useful to look at the invariance of the action. The action (4.1) is invariant under (4.2) and (4.3) if the following algebraic conditions

$$
J_{\nu}^{\mu}+K_{\nu}{ }^{\mu}=0, \quad L_{\mu \nu}=-L_{\nu \mu}, \quad P^{\mu \nu}=-P^{\nu \mu}
$$

as well as the differential conditions

$$
\frac{1}{2} P_{, \rho}^{\mu \nu}=-N_{\rho}^{\mu \nu}, \quad J_{[\nu, \rho]}^{\mu}=Q_{\nu \rho}^{\mu}, \quad \frac{1}{2} L_{[\mu \nu, \rho]}=M_{\rho \nu \mu}
$$

are satisfied. The differential conditions (4.6) allow us to express all three index tensors in terms of appropriate derivatives of two index tensors $J, P, L$ and $K$. These two index tensors can be combined as a single object

$$
\mathcal{J}=\left(\begin{array}{ll}
J & P \\
L & K
\end{array}\right)
$$

where $\mathcal{J}: T \oplus T^{*} \rightarrow T \oplus T^{*}$. It is easy to see that the algebraic part of the supersymmetry algebra (the part of (A.1,A.2) which does not involve derivatives nor three-index tensors) can be written as a single equation, namely that $\mathcal{J}^{2}=-1_{2 d}$. Passing then to the action, the algebraic condition (4.5) is equivalent to a hermiticity of $\mathcal{I}$ with respect to $\mathcal{J}$ (i.e., the natural pairing on $T \oplus T^{*}$, see previous Section). Therefore $\mathcal{J}$ is an almost generalized complex structure. Finally we have to analyze the eleven differential conditions coming from the algebra using (4.6). Using the results from the previous Section, we see that the three differential conditions arising from (A.1) are the same as the conditions (3.16)(3.18). The second line in (A.2) is equivalent to the condition (3.19). Surprisingly the remaining differential conditions in (A.2) are automatically satisfied provided that (3.16)(3.19) hold and $\mathcal{J}$ is a almost generalized complex structure. Therefore we have proved that the differential conditions that come from the supersymmetry algebra are equivalent to integrability of $\mathcal{J}$ with respect to the Courant bracket.

To summarize the topological model (4.1) admits $(2,0)$ supersymmetry if and only if manifold $\mathcal{M}$ is generalized complex manifold.

As we briefly mentioned in the previous Section, a generalized complex manifold is equivalent locally, via diffeomorphism and $b$-transform, to a product of a symplectic and a complex 
manifolds. If we choose the Darboux coordinates (label $n$ ) along the symplectic leaf and the standard complex coordinates (label $i, \bar{i}$ ) transverse to the leaf the supersymmetry transformations (4.2) and (4.3) is simplified drastically and have the following form

$$
\begin{array}{cc}
\delta \Phi^{i}=i \epsilon^{+} D_{+} \Phi^{i}, & \delta \Phi^{\bar{i}}=-i \epsilon^{+} D_{+} \Phi^{\bar{i}} \\
\delta S_{+i}=i \epsilon^{+} D_{+} S_{+i}, & \delta S_{+\bar{i}}=-i \epsilon^{+} D_{+} S_{+\bar{i}} \\
\delta \Phi^{n}=-\epsilon^{+} S_{+(n+1)}, & \delta S_{+(n+1)}=-i \epsilon^{+} \partial_{+} \Phi^{n} \\
\delta \Phi^{n+1}=\epsilon^{+} S_{+n}, & \delta S_{+n}=i \epsilon^{+} \partial_{+} \Phi^{n+1}
\end{array}
$$

\section{Topological model with WZ term}

In the previous Section we presented the topological model for which the extended supersymmetry is related to the generalized complex structure with integrability defined with the respect to the Courant bracket. The natural question is now the following: if in the integrability condition the Courant bracket is replaced by the twisted Courant bracket, can we then construct a model which incorporates twisted integrability? This is in fact possible and the solution is related to the WZ term.

We consider the topological model with an additional term

$$
S_{\text {top }}=\int d^{2} \sigma d \theta S_{+\mu} \partial_{=} \Phi^{\mu}-\frac{1}{2} \int d^{2} \sigma d \theta D_{+} \Phi^{\mu} \partial_{=} \Phi^{\nu} B_{\mu \nu}
$$

The last term is a WZ term and it depends only on a closed three-form $H$

$$
H_{\mu \nu \lambda}=\frac{1}{2}\left(B_{\mu \nu, \lambda}+B_{\lambda \mu, \nu}+B_{\nu \lambda, \mu}\right)
$$

if the world-sheet does not have a boundary. The model $(5.1)$ has $N=(1,0)$ supersymmetry and can be defined over any differential manifold $\mathcal{M}$ equipped with a closed three-form $H$. The Ansatz for the nonmanifest supersymmetry transformations is given by the same expressions as before, (4.2) and (4.3). The off-shell supersymmetry algebra is exactly the same, (4.4).

The main difference comes from the action. Namely invariance of the new action (5.1) under the transformations (4.2) and (4.3) leads to new relations between the three and two index tensors in the supersymmetry transformations. The action (5.1) is invariant under (4.2) and (4.3) if the following algebraic conditions are satisfied

$$
J_{\nu}^{\mu}+K_{\nu}{ }^{\mu}=0, \quad L_{\mu \nu}=-L_{\nu \mu}, \quad P^{\mu \nu}=-P^{\nu \mu}
$$


as well as the differential conditions

$$
\frac{1}{2} P_{, \rho}^{\mu \nu}=-N_{\rho}^{\mu \nu}, \quad J_{[\nu, \rho]}^{\mu}+P^{\mu \lambda} H_{\lambda \nu \rho}=Q_{\nu \rho}^{\mu}, \quad \frac{1}{2} L_{[\mu \nu, \rho]}+\frac{1}{2} J_{[\mu}^{\lambda} H_{\nu] \lambda \rho}=M_{\rho \nu \mu} .
$$

The algebraic part of all conditions remains the same as in the previous Section and therefore the two-index tensors can be combined in a single object $\mathcal{J}$ which is an almost generalized complex structure. However the differential conditions will change. Using (5.4) we have to require that the expressions (A.1) and (A.2) reproduce the supersymmetry algebra (4.4). Using the results from Section 3 we see that the three differential conditions arising from (A.1) are the same as conditions (3.25)-(3.27). The second line in (A.2) is equivalent to the condition (3.28). As before the remaining differential conditions in (A.2) are automatically satisfied provided that (3.25)-(3.28) hold and that $\mathcal{J}$ is a almost generalized complex structure. Therefore we have proved that the differential conditions coming from the supersymmetry algebra are equivalent to integrability of $\mathcal{J}$ with respect to the twisted Courant bracket.

\section{Sigma model}

Now we turn to the "real" sigma model. For the sake of clarity, let us assume that the WZ term is absent in the action. Thus the second order $N=(1,0)$ action is given by

$$
S=-i \int d^{2} \sigma d \theta D_{+} \Phi^{\mu} \partial_{=} \Phi^{\nu} E_{\mu \nu}(\Phi)
$$

This action has the following first order form

$$
S=i \int d^{2} \sigma d \theta\left(D_{+} \Phi^{\mu} S_{=\mu}-S_{+\mu} \partial_{=} \Phi^{\mu}-S_{+\mu} S_{=\nu} E^{\mu \nu}\right)
$$

Again, we would like to study under which conditions on the geometry of $\mathcal{M}$ the model (6.2) admits $(2,0)$ supersymmetry.

We start by giving the most general Ansatz for the nonmanifest supersymmetry transformations. We already gave the most general Ansatz for the transformations of $S_{+}^{\mu}$ and $\Phi^{\mu}$, see (4.2) and (4.3). For $S_{=}$we can write the following most general classical Ansatz for the transformations [12]

$$
\begin{array}{r}
\delta(\epsilon) S_{=\mu}=\epsilon^{+} D_{+} S_{=\nu} R_{\mu}^{\nu}+\epsilon^{+} \partial_{=} S_{+\nu} Z_{\mu}{ }^{\nu}+\epsilon^{+} D_{+} \partial_{=} \Phi^{\nu} T_{\mu \nu}+ \\
+\epsilon^{+} S_{+\rho} \partial_{=} \Phi^{\nu} U_{\mu \nu}{ }^{\rho}+\epsilon^{+} D_{+} \Phi^{\nu} S_{=\rho} V_{\mu \nu}{ }^{\rho}+\epsilon^{+} D_{+} \Phi^{\nu} \partial_{=} \Phi^{\rho} X_{\mu \nu \rho}+\epsilon^{+} S_{+\nu} S_{=\rho} Y_{\mu}{ }^{\nu \rho}
\end{array}
$$

Thus altogether the supersymmetry transformations contain 14 different tensors. The commutators of non-manifest supersymmetry transformations are given in Appendix. We have 
to require that (A.1) and (A.2) reduces to (4.4) (off-shell supersymmetry algebra) and that (A.3) reduces to

$$
\left[\delta\left(\epsilon_{2}\right), \delta\left(\epsilon_{1}\right)\right] S_{=\mu}=2 i \epsilon_{1}^{+} \epsilon_{2}^{+} \partial_{+} S_{=\mu}
$$

The action (6.2) is invariant under the transformations (4.2), (4.3) and (6.3) if the following algebraic conditions are satisfied

$$
\begin{aligned}
J_{\mu}^{\nu}+L_{\rho \mu} E^{\rho \nu}+R_{\mu}{ }^{\nu} & =0 \\
P^{\nu \mu}+E^{\rho \nu} K_{\rho}{ }^{\mu}+E^{\mu \rho} R_{\rho}{ }^{\nu} & =0 \\
L_{(\nu \mu)}+T_{(\mu \nu)} & =0 \\
Z_{\rho}{ }^{(\mu} E^{\nu) \rho}-P^{(\mu \nu)} & =0 \\
J_{\nu}^{\mu}+T_{\rho \nu} E^{\mu \rho}-Z_{\nu}{ }^{\mu}+K_{\nu}{ }^{\mu} & =0
\end{aligned}
$$

as well as the following differential conditions

$$
\begin{aligned}
J^{\mu}{ }_{[\nu, \rho]}-V_{[\nu \rho]}^{\mu}-M_{\lambda[\nu \rho]} E^{\lambda \mu}+R_{[\nu, \rho]}{ }^{\mu} & =0 \\
P_{, \rho}^{\mu \lambda}-E^{\lambda \nu} V_{\nu \rho}{ }^{\mu}-Y_{\rho}{ }^{\lambda \mu}-Q_{\nu \rho}{ }^{\lambda} E^{\nu \mu}+\left(E^{\lambda \nu} R_{\nu}{ }^{\mu}\right)_{, \rho}-E_{, \nu}^{\lambda \mu}{ }_{,{ }_{\rho}}^{\nu} & =0 \\
-U_{\lambda \mu}{ }^{\rho}-E^{\rho \nu} X_{\nu \lambda \mu}-Q_{\mu \lambda}{ }^{\rho}-J_{\lambda, \mu}^{\rho}+Z_{\lambda, \mu}^{\rho}-K_{\mu, \lambda}^{\rho}{ }_{\mu, \lambda} & =0 \\
X_{[\mu \lambda] \rho}+M_{\rho[\mu \lambda]}-\frac{1}{2} T_{[\mu \lambda], \rho}+L_{\rho[\mu, \lambda]}+\frac{1}{2} L_{[\mu \lambda], \rho} & =0 \\
\frac{1}{2}\left(Z_{\nu}{ }^{[\mu} E^{\rho] \nu}\right)_{, \lambda}-E^{[\mu \mid \nu} U_{\nu \lambda}{ }^{\mid \rho]}+N_{\lambda}{ }^{[\mu \rho]}+\frac{1}{2} P^{[\mu \rho]}{ }_{, \lambda} & =0 \\
-Y_{\nu}{ }^{[\lambda \mid \rho} E^{\mid \mu] \nu}+E^{\nu \rho} N_{\nu}{ }^{[\mu \lambda]}+E_{, \nu}^{[\mu \mid \rho} P^{\nu \mid \lambda]} & =0
\end{aligned}
$$

Combining these conditions with the supersymmetry algebra we may analyze the solutions of the problem. In particular we are interested in the geometrical interpretation of the solutions. We will see that to find a general solution is hard. This is partially due to absence of appropriate mathematical notions. However we will present the solution related to the generalized complex structure as defined by Hitchin [1]. Regarding more general solutions, we can offer only some speculations, presented in subsection 6.2.

Before turning to a discussion of possible solutions, we caution the reader that the general Ansatz we have made for the second supersymmetry will have solutions that correspond to "field equation"-type symmetries, as discussed in [12]. E.g., any transformation of the form

$$
\delta S_{+\mu}=\epsilon^{+} A_{\mu \nu} D_{+} F_{+}^{\nu}, \quad \delta S_{=\mu}=\epsilon^{+} D_{+}\left(A_{\nu \mu} F_{=}^{\nu}\right)
$$

will be a "trivial" symmetry of the $(2,0)$ action $(6.2)$ if $F_{+}^{\nu}$ and $F_{=}^{\nu}$ are the $S_{=\mu}$ and $S_{+\mu}$ field equations, respectively. 


\subsection{Algebraic conditions}

In this section we will analyze the content of the algebraic conditions coming from invariance of the action, (6.5-6.9), and from the algebraic part of the closure of the algebra, (A.1, A.2, A.3). For the topological model in section 4, we were able to reformulate all conditions in terms of an almost complex structure $\mathcal{J}$. Here we will try to get as close as we can to this doing the same for the sigma model, in particular we try to reexpress all conditions, now written in terms of $d \times d$ matrices, in terms of big $2 d \times 2 d$ matrices. The reason for this is to make contact with the generalized structures. In the case at hand, the geometry can even be analyzed in terms of the usual geometric structures on the manifold (and not on $T \oplus T^{*}$ ), analogously to the case dubbed "generalized Kähler structure" in [2]. (We will find the algebraic conditions of that case as an important particular case.)

We start by considering the conditions coming from the action. For example, equations $(6.5,6.6)$ can be written more elegantly as

$$
\left(\begin{array}{cc}
J^{t} & L^{t} \\
P^{t} & K^{t}
\end{array}\right)\left(\begin{array}{c}
E \\
1
\end{array}\right)=-\left(\begin{array}{c}
E \\
1
\end{array}\right) E^{-1} R E
$$

In what follows, we will refer to $d \times 2 d$ matrices such as the one in (6.17) as "vectors", so that the equation itself we can be thought of as the vector $\left(\begin{array}{c}E \\ 1\end{array}\right)$ being stabilized by the matrix $\mathcal{J}^{t}$, with "eigenvalue" $\left(-E^{-1} R E\right)$. If we define the projective action of $G L(2 d)$ on $d$-dimensional matrices as $\left(\begin{array}{ll}A & B \\ C & D\end{array}\right) \cdot E=(A E+B)(C E+D)^{-1}$, it is easy to eliminate the eigenvalue from (6.17), to find

$$
\mathcal{J}^{t} \cdot E=E
$$

In the old notation this equation reads $J^{t} E+L^{t}=E\left(P^{t} E+K^{t}\right)$, which means that $\mathcal{J}^{t}$ stabilizes $E$ under the projective action.

Turning to equations $(6.7,6.8,6.9)$, we put them in the form

$$
\widetilde{\mathcal{J}}^{t}+\mathcal{I} \widetilde{\mathcal{J}} \mathcal{I}=0, \quad \widetilde{\mathcal{J}} \equiv \mathcal{J}+\left(\begin{array}{c}
E^{-1} \\
1
\end{array}\right)(T,-Z)=\left(\begin{array}{cc}
J+E^{-1} T & P-E^{-1} Z \\
L+T & K-Z
\end{array}\right)
$$

Here $\mathcal{I}$ is again the metric $\left(\begin{array}{ll}0 & 1 \\ 1 & 0\end{array}\right)$ in (3.3). As explained there, the usual hermiticity condition for this metric reads $\mathcal{J}^{t} \mathcal{I} \mathcal{J}=\mathcal{I}$. Hence (6.19) is a hermiticity condition for $\widetilde{\mathcal{J}}$. We hasten to add that so far nothing says that this hermitian object squares to minus the identity, as was the case for $\mathcal{J}$ in the previous contexts. In fact, we shall see that in general this is not the case.

We now move to conditions coming from closure of the algebra. Fortunately, the algebraic parts in (A.1,A.2) were already analyzed in section 4. It is noticed there that they can be 
rewritten as the condition $\mathcal{J}^{2}=-1_{2 d}$. Condition (A.3) is harder and requires more care. Collecting the algebraic part gives the equations

$$
R Z+Z K-T P=0, \quad R T-Z L+T J=0, \quad R^{2}=-1 .
$$

The first two of these read more compactly

$$
(T,-Z)\left(\begin{array}{cc}
J & P \\
L & K
\end{array}\right)=-R(T,-Z) .
$$

Again, these condition can be thought of as a stabilization. As for the third condition in (6.20), we will show shortly that it is implied by the other conditions we already have.

(Before moving on, as a curiosity, we also notice that we can combine all of (6.20) with $\mathcal{J}^{2}=-1_{2 d}$, to give

$$
\left(\begin{array}{ccc}
R & T & -Z \\
0 & J & P \\
0 & L & K
\end{array}\right)^{2}=-1_{3 d}
$$

which thus summarizes all the algebraic equations from the algebra.)

We have now rewritten all conditions in ones that involve $2 d \times 2 d$ matrices. We use this to make contact with generalized structures. First of all, for the reader's convenience we list the algebraic conditions we have found:

1. $\mathcal{J}^{2}=-1_{2 d}($ from closure of the algebra, (A.1,A.2));

2. $\mathcal{J}^{t}\left(\begin{array}{c}E \\ 1\end{array}\right)=-\left(\begin{array}{c}E \\ 1\end{array}\right) E^{-1} R E(6.17)$;

3. $\mathcal{J}^{t}\left(\begin{array}{c}T^{t} \\ -Z^{t}\end{array}\right)=-\left(\begin{array}{c}T^{t} \\ -Z^{t}\end{array}\right) R^{t}(6.21)$;

4. $\widetilde{\mathcal{J}}+\mathcal{I} \widetilde{\mathcal{J}} \mathcal{I}=0$, where $\widetilde{\mathcal{J}} \equiv \mathcal{J}+\left(\begin{array}{c}E^{-1} \\ 1\end{array}\right)(T,-Z)$, eq. $(6.19)$

Having a list of objects on $T \oplus T^{*}$ and their conditions, it would seem natural at this point to ask to which subgroup of $\mathrm{SO}(d, d)$ they reduce. Unfortunately the conditions are not enough to determine a structure; there are many possible cases. This may be seen from the fact that conditions 2. and 3. may be more or less restrictive, depending on how many columns $\left(\begin{array}{c}T^{t} \\ -Z^{t}\end{array}\right)$ and $\left(\begin{array}{l}E \\ 1\end{array}\right)$ have in common. So they can range from $d$ to $2 d$ independent conditions.

To see this more explicitly, it is useful to change to a basis in which $\mathcal{J}$ simplifies. That this may be possible is again suggested by conditions 2 . and 3 . above: in the extreme case in which all columns of $\left(\begin{array}{c}T^{t} \\ -Z^{t}\end{array}\right)$ and $\left(\begin{array}{c}E \\ 1\end{array}\right)$ are all independent, they can be regarded as a basis 
in which $\mathcal{J}$ is block-diagonal. Rather than doing this, we will display another change of basis, which does not rely on any assumption about the rank of $\left(\begin{array}{cc}T^{t} E \\ -Z^{t} 1\end{array}\right)$.

The idea is to get another vector which is stabilized by $\mathcal{J}$, and which cannot have any column in common with one of those we already have, $\left(\begin{array}{c}E \\ 1\end{array}\right)$. The condition that this be stabilized, (6.17), implies indeed that also an orthogonal vector is stabilized:

$$
(1,-E) \mathcal{J}^{t}\left(\begin{array}{c}
E \\
1
\end{array}\right)=0
$$

This is seen to imply that

$$
(1,-E) \mathcal{J}^{t}=J_{-}^{t}(1,-E)
$$

for some $J_{-}$.

One might hope this result, along with (6.17), can be used to produce a block-diagonalizing change of basis. However, to do that we need both right actions or both left actions. But if we transpose (6.23), we get a statement on $\mathcal{J}$ and not $\mathcal{J}^{t}$. A way out of this situation would be to have a hermiticity condition related to $\mathcal{J}$; we do not have this, but the next best is condition 4. above, (6.19). ${ }^{7}$ Defining $X=\left(\begin{array}{c}E^{-1} \\ 1\end{array}\right)(T,-Z)$, this gives us

$\mathcal{J}^{t}\left(\begin{array}{c}-E^{t} \\ 1\end{array}\right)=-\left(\mathcal{I} \mathcal{J} \mathcal{I}+X+\mathcal{I} X^{t} \mathcal{I}\right)\left(\begin{array}{c}-E^{t} \\ 1\end{array}\right)=-\left(\left(\begin{array}{c}-E^{t} \\ 1\end{array}\right) J_{-}+\left(\begin{array}{c}1 \\ E^{-1}\end{array}\right)\left(Z E^{t}+T\right)\right)$.

With this further computation, and using the action of $\mathcal{J}$ on the other block-vector (6.17), we obtain

$$
\mathcal{J}^{t}=-\mathcal{I} \mathcal{E}\left(\begin{array}{cc}
E^{-1} R E & E^{-1} \theta \\
0 & J_{-}
\end{array}\right)(\mathcal{I E})^{-1}, \quad \mathcal{E} \equiv\left(\begin{array}{cc}
1 & 1 \\
E & -E^{t}
\end{array}\right), \quad \tau \equiv Z E^{t}+T .
$$

We have a basis in which $\mathcal{J}$ is block-triangular. Although we have not used condition 3 . yet, this form already shows that the stabilizer depends on the off-diagonal block $\tau$. Rather than to attempt a complete classification, we now show that the geometry can be described in terms of tensors on the manifold (which is not always the case in generalized complex geometry) and then return to the $T \oplus T^{*}$ point of view examining an important example.

The geometry can be analyzed in terms of tensors on the manifold for a simple reason. It is immediate to notice that the condition $\mathcal{J}^{2}=-1$ implies that

$$
R^{2}=-1, \quad J_{-}^{2}=-1, \quad R \tau=\tau J_{-} ;
$$

\footnotetext{
${ }^{7}$ Another possibility would have been that the hermitian object, $\mathcal{J}+X$, also squared to minus one. Unfortunately one finds $(\mathcal{J}+X)^{2}=-1+\mathcal{I} X^{t} \mathcal{I} X$, a relation similar to $\mathrm{U}(d)$ structures on manifolds of dimension higher than $d$.
} 
that is, $R$ and $J_{-}$are two almost complex structures, and $\tau$ is an intertwiner between them. (These facts could have been obtained without the change of basis; e.g., it is easy to show that condition 2. alone is enough to assume that the "eigenvalue" $R$ squares to minus one, and similarly from (6.23) for $J_{-}$.) The fact that $R$ squares to minus one also came more directly from the action, (of (6.20)); here we showed that it is a consequence of the other conditions 1.-4. above. This is why it was not included in that list.

We still have one condition that we have not used, 3. in the list above, equation (6.21). The condition is best analyzed after the $\mathcal{I E}$ change of basis. There, (6.21) reads

$$
\left(\begin{array}{cc}
E^{-1} R E & E^{-1} \tau \\
0 & J_{-}
\end{array}\right)\left(\begin{array}{c}
\zeta \\
-\frac{1}{2} g^{-1} \tau
\end{array}\right)=\left(\begin{array}{c}
\zeta \\
-\frac{1}{2} g^{-1} \tau
\end{array}\right) R^{t}
$$

$\zeta \equiv-\frac{1}{2} g^{-1}(T-Z E)^{t}$, and $\tau$ appears both in the matrix and in the vector, which makes the problem quadratic. Indeed, massaging the two components of these equations gives

$$
\tau\left(J_{-}-g^{-1} J_{-}^{t} g\right)=0, \quad R(E \zeta)-(E \zeta) R^{t}=\frac{1}{2} \tau g^{-1} \tau^{t} .
$$

These equations are modified (anti)-hermiticity properties on the two almost complex structures $R$ and $J_{-}$.

In summary, as seen from equations (6.25) and (6.27), there exists two almost complex structures, $R$ and $J_{-}$on the manifold, with an intertwiner between them, $\tau$; the two almost complex structures are antihermitian one on the image and one on the kernel of this intertwiner. Notice that $J_{-}$is equal to the almost complex structure of the usual sigma model (6.1) after integrating out the fields $S$ from the first order action (6.2).

\subsubsection{The hermitian case}

We now analyze an example, from both the $T \oplus T^{*}$ and the $T$ perspectives. Above, the problematic point was that the object which squares to minus one, $\mathcal{J}$, and the object which is hermitian, $\mathcal{J}+\left(\begin{array}{c}E^{-1} \\ 1\end{array}\right)(T,-Z)$, were not the same.

To overcome this, we assume in this subsection that $\mathcal{J}^{t}+\mathcal{I} \mathcal{J} \mathcal{I}=0$ (i.e., $\mathcal{J} \in O(d, d)$ ). Our previous formulae then reduce to the "generalized Kähler" geometry of [2], at least as far as algebraic conditions are concerned.

We start from the fact that $\mathcal{J}^{t}$ stabilizes $E=g+b$. Under the new hermiticity assumption, this is equivalent to the following statement:

$$
[\mathcal{J}, G]=0 .
$$


Here $G$ is a metric of signature $d, d$ defined as [2]

$$
G=\left(\begin{array}{ll}
-g^{-1} b & g^{-1} \\
g-b g^{-1} b & b g^{-1}
\end{array}\right)=-1_{2 d}+\left(\begin{array}{l}
1 \\
E
\end{array}\right) g^{-1}\left(\begin{array}{ll}
E^{t} & 1
\end{array}\right)
$$

with the property that $G^{2}=1_{2 d}$ and $G^{t} \mathcal{I} G=\mathcal{I}$. $G$ (or $E$ ) reduces the structure group on $T \oplus T^{*}$ to $\mathrm{O}(d) \times \mathrm{O}(d)$. $\mathcal{J}$ reduces the structure to $\mathrm{U}(d / 2, d / 2)$. Together, and with the compatibility condition (6.28) ( or $\mathcal{J} \cdot E=E$ ), they reduce to $\mathrm{U}(d / 2) \times \mathrm{U}(d / 2)$.

Equation (6.28) can be shown formally from the stabilization condition, but is particularly easy to see in the basis introduced above. From (6.24) and (6.29), one gets

$$
\mathcal{J}=\mathcal{E}\left(\begin{array}{cc}
E^{-1} R E & \\
0 & J_{-}
\end{array}\right) \mathcal{E}^{-1}, \quad G=\mathcal{E}\left(\begin{array}{cc}
1 & \\
0 & -1
\end{array}\right) \mathcal{E}^{-1}, \quad I=\mathcal{E}\left(\begin{array}{ll}
g & \\
0 & -g
\end{array}\right) \mathcal{E}^{-1}
$$

The first equation in (6.30) is the same as equation (6.3) in [2], after redefining $J_{+} \equiv E^{-1} R E$. (There, the change of basis $\mathcal{E}$ has been factorized as $\left(\begin{array}{ll}1 & 0 \\ b & 1\end{array}\right)\left(\begin{array}{ll}1 & 1 \\ g & -g\end{array}\right)$.) As for the general case, $J_{ \pm}$ are almost complex structures. However, given the hermiticity assumption, the form of the pairing $\mathcal{I}$ in (6.30) also shows that these two almost complex structures are both hermitian with respect to the metric $g$. This geometry is called (almost) bi-hermitian on the manifold.

We are not done yet, because imposing hermiticity does not set $T$ and $Z$ to zero. What one gets is the remnant of (6.19), that is, $X+\mathcal{I} X^{t} \mathcal{I}$. In components, this gives $T^{t}=-T$ and $Z^{t}=E^{-1} T$. Using condition 3. yields $R T^{t}-T R^{t}=0$, hence, $T$ is an intertwiner between $R$ and its transpose. Equivalently, we might want to define the matrix

$$
\hat{\mathcal{J}}=\left(\begin{array}{ll}
R^{t} & 0 \\
T & -R
\end{array}\right)
$$

which is then an almost generalized complex structure.

It is also interesting to see what happens if we slightly relax the initial condition. Looking at the triangular form for $\mathcal{J},(6.24)$, a natural condition is $\tau=0$. ( $\tau$ is only one component of $X+\mathcal{I} X^{t} \mathcal{I}$, and thus this is weaker than the hermiticity considered above.) In this case, we have a condition similar to (6.28), namely $\left[I \mathcal{J}^{t} I, G\right]=0$. We still have a reduction to $\mathrm{U}(d / 2) \times \mathrm{U}(d / 2)$. And we still have the two almost complex structures (we even had them in the general case). But, since we have no hermiticity, $J_{ \pm}$will no longer be hermitian with respect to the same metric $g$.

\subsection{Differential conditions}

In this subsection we discuss the differential conditions which arise both from invariance of the action and from the supersymmetry algebra. We are unable to solve the problem 
completely. The difficulties in finding the geneal solution may be partially ascribed to a lack of the appropriate mathematical tools.

As discussed in subsection 6.1, even at the algebraic level the natural object $\mathcal{J}$ does not fit into the Hitchin framework unless extra restrictions are imposed, but setting $8=0$ and $T=0$ (as in the solution just discussed) leads to $\mathcal{J}$ being an almost generalized complex structure. We consider only the case when $T=Z=0$.

With the differential conditions the situation is very similar. If we impose extra restrictions by hand then we may ensure that $\mathcal{J}$ is a generalized complex structure. E.g., imposing $X_{\mu \nu \lambda}=0$ and $U_{\mu \nu}{ }^{\rho}=0$ (again as in the solution above) we find that the conditions (6.12)(6.14) coincide with the conditions (4.6), for the topological model. Therefore we can use the results from Section 4 and conclude that the supersymmetry algebra (A.1) and (A.2) together with (6.12)-(6.14) implies that $\mathcal{J}$ is a generalized complex structure. The remaining constraints that come from the invariance of the action (6.10), (6.11) and (6.15), can be rewritten as

$$
\begin{array}{r}
V_{[\nu \rho]}^{\mu}=L_{\sigma \rho} E_{, \nu}^{\sigma \mu}+L_{\nu \sigma} E_{, \rho}^{\sigma \mu}-L_{\rho \nu, \sigma} E^{\sigma \mu} \\
E^{\lambda \nu} V_{\nu \rho}{ }^{\mu}+Y_{\rho}{ }^{\lambda \mu}=E^{\nu \mu}{ }_{, \rho} J^{\lambda}{ }_{\nu}+J_{\rho, \nu}^{\lambda} E^{\nu \mu}-E^{\lambda \mu}{ }_{, \nu} J_{\rho}{ }_{\rho} \\
Y_{\nu}{ }^{[\lambda \mid \rho} E^{\mid \mu] \nu}=E_{, \nu}^{\mu \rho}{ }^{\nu \lambda}-E^{\lambda \rho}{ }_{, \nu} P^{\nu \mu}-E^{\nu \rho} P^{\mu \lambda}{ }_{, \nu}
\end{array}
$$

and there are eight non-trivial conditions from the algebra for $S_{=}$, (A.3). From (6.32)-(6.34) we derive the following differential condition for $\mathcal{J}$ and $E$

$$
\begin{array}{r}
E^{\lambda \nu} E^{\gamma \rho}\left(L_{\sigma \rho} E_{, \nu}^{\sigma \mu}+L_{\nu \sigma} E_{, \rho}^{\sigma \mu}-L_{\rho \nu, \sigma} E^{\sigma \mu}\right)+E_{, \nu}^{\gamma \mu} P^{\nu \lambda}-E_{, \nu}^{\lambda \mu} P^{\nu \gamma}-E^{\nu \mu} P_{, \nu}^{\gamma \lambda}= \\
=E^{[\gamma \mid \rho} E_{, \rho}^{\nu \mu} J_{\nu}^{\mid \lambda]}+J_{\rho, \nu}^{[\lambda} E^{\gamma] \rho} E^{\nu \mu}-E_{, \nu}^{[\lambda \mid \mu} E^{\mid \gamma] \rho} J_{\rho}^{\nu}
\end{array}
$$

which resembles a condition for the complex structure to be covariantly constant. This is indeed the interpretation for the solutions presented below.

To summarize, the generalized sigma model (6.2) admits $(2,0)$ supersymmetry (4.2), (4.3) and (6.3) (with $T=Z=X=U=0$ ) if on $\mathcal{M}$ there exists a generalized complex structure $\mathcal{J}$ such (6.18) and a number of differential conditions is satisfied.

Although we cannot offer an interpretation of these differential conditions in geometrical terms, it is not hard to construct additional specific examples. The main problem comes from the $S_{=}$algebra. However if we assume that $R$ is a complex structure, then there exists the coordinates when $R$ is constant and $Y=V=0$. These assumptions do solve the $S_{=}$ algebra (A.3), but this is not the most general solution. Using this observation we may

\footnotetext{
${ }^{8}$ In general it is enough to put $Z^{t}=E^{-1} T$. However for the sake of clarity we discuss only the solution $T=Z=0$.
} 
construct various examples. We start from the simplest case with a diagonal generalized complex structure $\mathcal{J}$ (i.e., $P=L=0$ ). In this case $J^{t}+R=0$ and we may use complex coordinates (the same for $J$ and $R$ ) and assume $Y=V=0$ in this coordinates. Thus the supersymmetry algebra is automatically satisfied. From (6.18) we obtain that $E=J^{t} E J$ and thus $E$ is a $(1,1)$ tensor with respect to $J$. The remaining condition (6.35) implies that $E_{i \bar{k}, j}-E_{j \bar{k}, i}=0$, which says that $J$ is covariantly constant with respect to a connection with the torsion $H=d b$.

There exists a different way of looking for solutions. We present a solution based on two reasonable assumptions. The $N=(2,0)$ action (6.2) has a discrete symmetry analogous to that discussed in [12] for the $N=(2,2)$ model. It is invariant under

$$
S_{+\mu} \rightarrow-S_{+\mu}+2 D_{+} \Phi^{\lambda} E_{\lambda \mu} \quad S_{=\mu} \rightarrow-S_{=\mu}-2 \partial_{=} \Phi^{\lambda} E_{\mu \lambda}
$$

Our first assumption is that the symmetry (6.36) commutes with the second supersymmetry. This yields nine conditions on the parameter fields, four of which are

$$
P^{\mu \nu}=0, N_{\mu}^{\nu \rho}=0, Y_{\mu}^{\nu \rho}=0, U_{\nu \rho}^{\mu}=0
$$

With the additional requirement that $K=-J^{t}$, we solve all conditions, algebraic as well as differential. We find that $J$ is a complex structure which is covariantly constant with respect to the +-connection. I.e. writing $J_{\mu \nu}=J_{\rho}^{\mu} g_{\rho \nu}$ where the hermitean metric $g_{\mu \nu} \equiv \frac{1}{2} E_{(\mu \nu)}$, we have

$$
\nabla_{\rho}^{(+)} J_{\mu \nu}=\partial_{\rho} J_{\mu \nu}-\left(\Gamma_{\rho[\mu \mid \tau}^{(0)}+H_{\rho[\mu \mid \tau}\right) J_{\nu]}^{\tau}=0,
$$

where $\Gamma^{(0)}$ is the Levi-Civita connection for $g_{\mu \nu}$ and the torsion is the three-form $H=d b$. The rest of the solution is given in terms of $J, E$ and $b$ according to:

$$
\begin{aligned}
& L_{\mu \nu}=\frac{1}{2} L_{[\mu \nu]}=J_{[\nu}^{\rho} b_{\mu] \rho} \\
& R_{\mu}{ }^{\nu}=E_{\mu \rho} J_{\tau}^{\rho} E^{\tau \nu} \\
& 2 M_{\mu \nu \rho}=L_{[\nu \mu \rho]} \\
& V_{\mu \nu}{ }^{\sigma}=E_{\mu \lambda}\left(J^{\lambda}{ }_{\nu, \tau} E^{\tau \sigma}+J_{\tau}^{\lambda} E_{, \nu}^{\tau \sigma}-E_{, \tau}^{\lambda \sigma} J_{\nu}^{\tau}\right) \\
& Q_{\mu \nu}{ }^{\sigma}=J^{\sigma}{ }_{[\mu, \nu]} .
\end{aligned}
$$

In addition

$$
T_{\mu \nu}=0, Z_{\mu}^{\nu}=0, X_{\mu \nu \rho}=0 .
$$

This solution may be recast in different forms using (6.38). In the first example, $b \in \Omega^{1,1}(\mathcal{M})$. Above we have analyzed the situation when $b^{2,0}$ and $b^{0,2}$ are allowed and the generalized complex structure has the form

$$
\mathcal{J}=\left(\begin{array}{cc}
J & 0 \\
L & -J^{t}
\end{array}\right)
$$


where $L$ is a $(2,0)$ and $(0,2)$ tensor with respect to the complex structure $J$, such that $L_{i j}=2 b_{i j}$. The metric $g$ is hermitian with respect to $J$. The integrability of $\mathcal{J}$ implies that $\partial L^{2,0}=0$.

Analogously we consider the following generalized complex structure

$$
\mathcal{J}=\left(\begin{array}{cc}
J & P \\
0 & -J^{t}
\end{array}\right)
$$

where $P$ is a $(2,0)$ contravariant tersor with respect to the complex structure $J$ (and $J^{t}+R=$ $0)$. $P$ is proportional to $(2,0)$ part of $\theta$ (antisymmetric part of $E^{-1}$ ). Again the differential condition (6.35) can be understood as an appropriate covariantly constancy condition for $J$.

These examples are all realized on a complex manifold $\mathcal{M}$. We do not know if a generic solution is always a complex manifold. Notice that, in the first order model we have (incompletely) analyzed, there are more tensors in the game than in the second order model. Due to this, there are many more subcases that can be considered.

\section{Summary}

In this paper our aim was to find a world-sheet realization of the generalized complex structure recently introduced by Hitchin. We have considered three different two dimensional models inspired by the first order action for the standard sigma model. The main property of these models is that the fields take values in $T \oplus T^{*}$. We have found that the extended supersymmetry for these models is closely related to the generalized complex structure. This is the main result of the paper.

We have left many unanswered questions and open problems. E.g., we were unable to find a geometrical interpretation for a generic $(2,0)$ generalized sigma model. In general the main problem is that $\mathcal{J}$ does not respect the natural paring on $T \oplus T^{*}$. Presumably one needs to introduce a more general Courant algebroid on $T \oplus T^{*}$ related to a different paring. We did not consider in detail certain models which appears naturally in the present context: the supersymmetric Poisson sigma model (i.e., when $E$ is Poisson structure), the supersymmetric Poisson-WZ model and the generalized sigma model with WZ term. Many statements from this paper can be easily extended to these models.

Finally, and maybe most unsatisfyingly, we were not able to show the possibility of having non-complex manifolds as supersymmetric backgrounds for our model (which would have been a powerful motivation for the present paper), while not being able to rule it out either. A reason for the technical complication we are facing for the "physical" first 
order sigma model may be related to its non-uniqueness (i. e., we could have taken other combinations of the first and second order action); maybe there are choices which make the equations simpler to solve. Independently from this, at the present level of development of the formalism, there are intrinsic technical difficulties coming for example from a big number of second and third-order tensors; presumably some better formalism to tackle with them will be needed for further progress.

Acknowledgements: The work of UL is supported in part by VR grant 650-1998368. The work of RM and AT is supported in part by EU contract HPRN-CT-2000-00122 and by INTAS contracts 55-1-590 and 00-0334. We are grateful to Marco Gualtieri, Simeon Hellerman, Nigel Hitchin, Daniel Huybrechts and Pierre Vanhove for intersting discussions.

\section{A Appendix}

Through the paper we use the following conventions

$$
A_{[\mu \nu]}=A_{\mu \nu}-A_{\nu \mu}, \quad A_{(\mu \nu)}=A_{\mu \nu}+A_{\nu \mu}, \quad L_{[\mu \nu, \rho]}=L_{\mu \nu, \rho}+L_{\rho \mu, \nu}+L_{\nu \rho, \mu}
$$

where $L$ is antisymmetric.

Below we give the complete expressions for the commutators of nonmanifest supersymmetry acting on all fields.

$$
\begin{aligned}
& {\left[\delta\left(\epsilon_{2}\right), \delta\left(\epsilon_{1}\right)\right] \Phi^{\mu}=-2 i \epsilon_{1}^{+} \epsilon_{2}^{+} \partial_{+} \Phi^{\lambda}\left(J^{\mu}{ }_{\nu} J_{\lambda}^{\nu}+P^{\mu \nu} L_{\nu \lambda}\right)+2 \epsilon_{1}^{+} \epsilon_{2}^{+} D_{+} S_{+\lambda}\left(J_{\nu}^{\mu} P^{\nu \lambda}+P^{\mu \nu} K_{\nu}{ }^{\lambda}\right)+} \\
& +2 \epsilon_{1}^{+} \epsilon_{2}^{+} D_{+} \Phi^{\lambda} D_{+} \Phi^{\rho}\left(J_{\lambda, \rho}^{\nu} J_{\nu}^{\mu}-J_{\lambda, \nu}^{\mu} J_{\rho}^{\nu}-M_{\nu \lambda \rho} P^{\mu \nu}\right)+2 \epsilon_{1}^{+} \epsilon_{2}^{+} S_{+\lambda} S_{+\rho}\left(P_{, \nu}^{\mu \rho} P^{\nu \lambda}-P^{\mu \nu} N_{\nu}^{\lambda \rho}\right)+ \\
& +2 \epsilon_{1}^{+} \epsilon_{2}^{+} D_{+} \Phi^{\nu} S_{+\lambda}\left(J_{\nu, \rho}^{\mu} P^{\rho \lambda}+P_{, \nu}^{\rho \lambda} J_{\rho}^{\mu}-Q_{\rho \nu}^{\lambda} P^{\mu \rho}-P_{, \rho}^{\mu \lambda} J_{\nu}^{\rho}\right) \\
& {\left[\delta\left(\epsilon_{2}\right), \delta\left(\epsilon_{1}\right)\right] S_{+\mu}=-2 i \epsilon_{1}^{+} \epsilon_{2}^{+} \partial_{+} S_{+\lambda}\left(K_{\mu}{ }^{\nu} K_{\nu}{ }^{\lambda}+L_{\mu \nu} P^{\nu \lambda}\right)+2 i \epsilon_{1}^{+} \epsilon_{2}^{+} \partial_{+} D_{+} \Phi^{\lambda}\left(K_{\mu}{ }^{\nu} L_{\nu \lambda}+L_{\mu \nu} J_{\lambda}^{\nu}\right)+} \\
& +2 i \epsilon_{1}^{+} \epsilon_{2}^{+} D_{+} \Phi^{\lambda} \partial_{+} \Phi^{\rho}\left(L_{\mu \nu} J_{\lambda, \rho}^{\nu}+J_{\lambda}^{\nu} L_{\mu \rho, \nu}+K_{\mu}{ }^{\nu} L_{\nu \rho, \lambda}+2 K_{\mu}{ }^{\nu} M_{\nu \rho \lambda}-2 J_{\rho}^{\nu} M_{\mu \nu \lambda}-Q_{\mu \lambda}{ }^{\nu} L_{\nu \rho}\right)+ \\
& +2 i \epsilon_{1}^{+} \epsilon_{2}^{+} S_{+\lambda} \partial_{+} \Phi^{\rho}\left(-P_{, \rho}^{\nu \lambda} L_{\mu \nu}-L_{\mu \rho, \nu} P^{\nu \lambda}+Q_{\nu \rho}{ }^{\lambda} K_{\mu}{ }^{\nu}+2 L_{\nu \rho} N_{\mu}{ }^{\nu \lambda}-J_{\rho}^{\nu} Q_{\mu \nu}{ }^{\lambda}\right)+ \\
& +2 \epsilon_{1}^{+} \epsilon_{2}^{+} D_{+} S_{+\lambda} D_{+} \Phi^{\rho}\left(-K_{\nu, \rho}{ }^{\lambda} K_{\mu}{ }^{\nu}-Q_{\nu \rho}{ }^{\lambda} K_{\mu}{ }^{\nu}-K_{\mu, \nu}{ }^{\lambda} J_{\rho}^{\nu}+2 P^{\nu \lambda} M_{\mu \nu \rho}+Q_{\mu \rho}{ }^{\nu} K_{\nu}{ }^{\lambda}\right)+ \\
& +2 \epsilon_{1}^{+} \epsilon_{2}^{+} D_{+} \Phi^{\lambda} D_{+} \Phi^{\rho} D_{+} \Phi^{\gamma}\left(M_{\nu \lambda \rho, \gamma} K_{\mu}{ }^{\nu}-2 J_{\lambda, \gamma}^{\nu} M_{\mu \nu \rho}+M_{\mu \lambda \rho, \nu} J_{\gamma}^{\nu}+Q_{\mu \rho}{ }^{\nu} M_{\nu \lambda \gamma}\right)+ \\
& +2 \epsilon_{1}^{+} \epsilon_{2}^{+} D_{+} S_{+\lambda} S_{+\rho}\left(2 N_{\nu}{ }^{\lambda \rho} K_{\mu}{ }^{\nu}+K_{\mu}{ }^{\lambda}{ }_{, \nu} P^{\nu \rho}-2 K_{\nu}{ }^{\lambda} N_{\mu}{ }^{\nu \rho}+P^{\nu \lambda} Q_{\mu \nu}{ }^{\rho}\right)+ \\
& +2 \epsilon_{1}^{+} \epsilon_{2}^{+} S_{+\lambda} S_{+\rho} D_{+} \Phi^{\gamma}\left(N_{\nu}^{\lambda \rho}{ }_{, \gamma} K_{\mu}{ }^{\nu}+2 Q_{\nu \gamma}{ }^{\lambda} N_{\mu}{ }^{\nu \rho}+N_{\mu}^{\lambda \rho}{ }_{, \nu} J_{\gamma}^{\nu}+P_{, \gamma}^{\nu \lambda} Q_{\mu \nu}{ }^{\rho}-Q_{\mu \gamma}{ }^{\nu} N_{\nu}{ }^{\lambda \rho}-\right. \\
& \left.-Q_{\mu \gamma}{ }^{\lambda}{ }_{, \nu} P^{\nu \rho}\right)+2 \epsilon_{1}^{+} \epsilon_{2}^{+} D_{+} \Phi^{\rho} S_{+\lambda} D_{+} \Phi^{\gamma}\left(Q_{\nu \rho}{ }^{\lambda}{ }_{, \gamma} K_{\mu}{ }^{\nu}-2 M_{\nu \rho \gamma} N_{\mu}{ }^{\nu \lambda}-2 P^{\nu \lambda}{ }_{, \gamma} M_{\mu \nu \rho}-M_{\mu \gamma \rho, \nu} P^{\nu \lambda}-\right. \\
& \left.-J_{\rho, \gamma}^{\nu} Q_{\mu \nu}{ }^{\lambda}+Q_{\mu \rho}{ }^{\nu} Q_{\nu \gamma}{ }^{\lambda}+Q_{\mu \rho, \nu}{ }^{\lambda} J_{\gamma}^{\nu}\right)+2 \epsilon_{1}^{+} \epsilon_{2}^{+} S_{+\lambda} S_{+\gamma} S_{+\rho}\left(2 N_{\nu}{ }^{\lambda \gamma} N_{\mu}{ }^{\nu \rho}-N_{\mu}{ }^{\lambda \gamma}{ }_{, \nu} P^{\nu \rho}\right)
\end{aligned}
$$




$$
\begin{aligned}
& {\left[\delta\left(\epsilon_{2}\right), \delta\left(\epsilon_{1}\right)\right] S_{=\mu}=-2 \epsilon_{1}^{+} \epsilon_{2}^{+}\left[i \partial_{+} S_{=\rho}\left(R_{\nu}{ }^{\rho} R_{\mu}{ }^{\nu}\right)+\right.} \\
& +D_{+} \partial_{=} S_{+\rho}\left(Z_{\nu}{ }^{\rho} R_{\mu}{ }^{\nu}+K_{\nu}{ }^{\rho} Z_{\mu}{ }^{\nu}-P^{\nu \rho} T_{\mu \nu}\right)+i \partial_{+} \partial_{=} \Phi^{\rho}\left(T_{\nu \rho} R_{\mu}{ }^{\nu}-L_{\nu \rho} Z_{\mu}{ }^{\nu}+J_{\rho}^{\nu} T_{\mu \nu}\right)+ \\
& +D_{+} S_{+\nu} \partial_{=} \Phi^{\rho}\left(U_{\sigma \rho}{ }^{\nu} R_{\mu}{ }^{\sigma}+K_{\sigma, \rho}{ }^{\nu} Z_{\mu}{ }^{\sigma}-P^{\sigma \nu}{ }_{, \rho} T_{\mu \sigma}+K_{\sigma}{ }^{\nu} U_{\mu \rho}{ }^{\sigma}-P^{\sigma \nu} X_{\mu \sigma \rho}\right)+ \\
& +S_{+\nu} D_{+} \partial_{=} \Phi^{\rho}\left(-U_{\sigma \rho}{ }^{\nu} R_{\mu}{ }^{\sigma}+Q_{\sigma \rho}{ }^{\nu} Z_{\mu}{ }^{\sigma}+T_{\mu \rho, \sigma} P^{\sigma \nu}+P_{, \rho}^{\sigma \nu} T_{\mu \sigma}+J_{\rho}^{\sigma} U_{\mu \sigma}{ }^{\nu}+T_{\sigma \rho} Y_{\mu}{ }^{\nu \sigma}\right)+ \\
& +S_{+\nu} \partial_{=} \Phi^{\rho} D_{+} \Phi^{\sigma}\left(-U_{\lambda \rho}{ }_{, \sigma}^{\nu} R_{\mu}{ }^{\lambda}+Q_{\lambda \sigma}{ }^{\nu}{ }_{, \rho} Z_{\mu}{ }^{\lambda}+X_{\mu \sigma \rho, \lambda} P^{\lambda \nu}+P_{, \rho \sigma}^{\lambda \nu} T_{\mu \lambda}+Q_{\lambda \sigma}{ }^{\nu} U_{\mu \rho}{ }^{\lambda}+\right. \\
& \left.+U_{\mu \rho}^{\nu}{ }_{, \lambda} J_{\sigma}^{\lambda}+J_{\sigma, \rho}^{\lambda} U_{\mu \lambda}{ }^{\nu}-P_{, \sigma}^{\lambda \nu} X_{\mu \lambda \rho}+P_{, \rho}^{\lambda \nu} X_{\mu \sigma \lambda}+X_{\lambda \sigma \rho} Y_{\mu}{ }^{\nu \lambda}-U_{\lambda \rho}{ }^{\nu} V_{\mu \sigma}{ }^{\lambda}\right)+ \\
& +i \partial_{+} \Phi^{\nu} S_{=\rho}\left(V_{\lambda \nu}{ }^{\rho} R_{\mu}{ }^{\lambda}-L_{\lambda \nu} Y_{\mu}^{\lambda \rho}+J^{\sigma}{ }_{\nu} V_{\mu \sigma}{ }^{\rho}\right)+D_{+} \Phi^{\nu} D_{+} S_{=\rho}\left(-V_{\lambda \nu}{ }^{\rho} R_{\mu}{ }^{\lambda}-R_{\mu}{ }^{\rho}{ }_{\lambda, \lambda} J_{\nu}^{\lambda}+\right. \\
& \left.+R_{\sigma}{ }^{\rho} V_{\mu \nu}{ }^{\sigma}+R_{\sigma, \nu}{ }^{\rho} R_{\mu}{ }^{\sigma}\right)+D_{+} \Phi^{\nu} D_{+} \Phi^{\rho} S_{=\sigma}\left(V_{\lambda \rho}{ }^{\sigma}{ }_{\nu} R_{\mu}{ }^{\lambda}+V_{\mu \nu}{ }^{\sigma}{ }_{\lambda} J^{\lambda}{ }_{\rho}-M_{\lambda \nu \rho} Y_{\mu}{ }^{\lambda \sigma}+\right. \\
& \left.+J_{\rho, \nu}^{\lambda} V_{\mu \lambda}{ }^{\sigma}+V_{\lambda \rho}{ }^{\sigma} V_{\mu \nu}{ }^{\lambda}\right)+D_{+} S_{+\nu} S_{=\rho}\left(Y_{\sigma}{ }^{\nu \rho} R_{\mu}^{\sigma}+K_{\sigma}{ }^{\nu} Y_{\mu}{ }^{\sigma \rho}-P^{\sigma \nu} V_{\mu \sigma}{ }^{\rho}\right)+ \\
& +S_{+\nu} D_{+} S_{=\rho}\left(-Y_{\sigma}{ }^{\nu \rho} R_{\mu}^{\sigma}+R_{\mu}{ }^{\rho}{ }_{, \lambda} P^{\lambda \nu}+R_{\sigma}{ }^{\rho} Y_{\mu}{ }^{\nu \sigma}\right)+ \\
& +S_{+\nu} S_{=\rho} D_{+} \Phi^{\sigma}\left(-Y_{\lambda}{ }_{, \sigma}^{\nu \rho} R_{\mu}{ }^{\lambda}+V_{\mu \sigma}{ }^{\rho}{ }_{, \lambda} P^{\lambda \nu}+Y_{\mu}{ }^{\nu \rho}{ }_{\lambda} J^{\lambda}{ }_{\sigma}+Q_{\lambda \sigma}{ }^{\nu} Y_{\mu}{ }^{\lambda \rho}+V_{\lambda \sigma}{ }^{\rho} Y_{\mu}{ }^{\nu \lambda}+P_{, \sigma}^{\lambda \nu} V_{\mu \lambda}{ }^{\rho}-\right. \\
& \left.-Y_{\lambda}{ }^{\nu \rho} V_{\mu \sigma}{ }^{\lambda}\right)+i \partial_{+} \Phi^{\nu} \partial_{=} \Phi^{\rho}\left(-L_{\sigma \nu, \rho} Z_{\mu}{ }^{\sigma}+{J^{\sigma}}_{\nu, \rho} T_{\mu \sigma}-L_{\sigma \nu} U_{\mu \rho}{ }^{\sigma}+J^{\sigma}{ }_{\nu} X_{\mu \sigma \rho}+X_{\sigma \nu \rho} R_{\mu}{ }^{\sigma}\right)+ \\
& +\partial_{=} S_{+\nu} S_{+\rho}\left(-2 N_{\sigma}{ }^{[\nu \rho]} L_{\mu}{ }^{\sigma}-Z_{\mu}{ }_{, \sigma}^{\nu} P^{\sigma \rho}+P^{\sigma \nu} U_{\mu \sigma}{ }^{\rho}-Z_{\sigma}{ }^{\nu} Y_{\mu}{ }^{\rho \sigma}\right)+ \\
& +S_{+\nu} S_{+\rho} \partial_{=} \Phi^{\sigma}\left(-N_{\lambda}{ }^{\nu \rho}{ }_{, \sigma} Z_{\mu}^{\lambda}+U_{\mu \sigma}{ }_{, \lambda}{ }^{\lambda} P^{\lambda \nu}-N_{\lambda}{ }^{\nu \rho} U_{\mu \sigma}{ }^{\lambda}-P_{, \sigma}^{\lambda \rho} U_{\mu \lambda}{ }^{\nu}+U_{\lambda \sigma}{ }^{\rho} Y_{\mu}{ }^{\nu \lambda}\right)+ \\
& +D_{+} \partial_{=} \Phi^{\nu} D_{+} \Phi^{\rho}\left(-2 M_{\sigma[\nu \rho]} Z_{\mu}{ }^{\sigma}+T_{\mu \nu, \lambda} J_{\rho}^{\lambda}+2 J_{[\rho, \nu]}^{\lambda} T_{\mu \lambda}-J_{\nu}^{\sigma} X_{\mu \rho \sigma}+X_{\sigma \rho \nu} R_{\mu}^{\sigma}-T_{\sigma \nu} V_{\mu \rho}{ }^{\sigma}-\right. \\
& \left.-T_{\sigma \nu, \rho} R_{\mu}^{\sigma}\right)+D_{+} \Phi^{\nu} D_{+} \Phi^{\rho} \partial_{=} \Phi^{\sigma}\left(-M_{\lambda \nu \rho, \sigma} Z_{\mu}{ }^{\lambda}-X_{\mu \rho \sigma, \lambda} J_{\nu}^{\lambda}-J_{\nu, \rho \sigma}^{\lambda} T_{\mu \lambda}-M_{\lambda \nu \rho} U_{\mu \sigma}{ }^{\lambda}-\right. \\
& \left.-J_{\nu, \rho}^{\lambda} X_{\mu \lambda \sigma}+J_{\rho, \sigma}^{\lambda} X_{\mu \nu \lambda}+X_{\lambda \rho \sigma, \nu} R_{\mu}^{\lambda}+X_{\lambda \rho \sigma} V_{\mu \nu}{ }^{\lambda}\right)-D_{+} \Phi^{\nu} \partial_{=} S_{+\rho}\left(Q_{\lambda \nu}{ }^{\rho} Z_{\mu}{ }^{\lambda}+Z_{\mu}{ }^{\rho}{ }_{, \lambda} J^{\lambda}{ }_{\nu}+\right. \\
& \left.+P_{, \nu}^{\lambda \rho} T_{\mu \lambda}+P^{\lambda \rho} X_{\mu \nu \lambda}-Z_{\sigma}{ }^{\rho} V_{\mu \nu}{ }^{\sigma}-Z_{\sigma}{ }^{\rho}{ }_{, \nu} R_{\mu}{ }^{\sigma}\right)+S_{+\nu} S_{+\rho} S_{=\sigma}\left(Y_{\mu}{ }^{\rho \sigma}{ }_{, \lambda} P^{\lambda \nu}-\right. \\
& \left.\left.-N_{\lambda}{ }^{\nu \rho} Y_{\mu}{ }^{\lambda \sigma}+Y_{\lambda}{ }^{\rho \sigma} Y_{\mu}{ }^{\nu \lambda}\right)\right] \text {. }
\end{aligned}
$$

\section{References}

[1] N. Hitchin, "Generalized Calabi-Yau manifolds," Q. J. Math. 54 (2003), no. 3, 281308, arXiv:math.DG/0209099.

[2] M. Gualtieri, "Generalized complex geometry," Oxford University DPhil thesis, arXiv:math.DG/0401221.

[3] D. Huybrechts, "Generalized Calabi-Yau structures, K3 surfaces, and B-fields," arXiv:math.AG/0306162.

[4] A. Kapustin and D. Orlov, "Vertex algebras, mirror symmetry, and D-branes: The case of complex tori," Commun. Math. Phys. 233 (2003) 79 [arXiv:hep-th/0010293]. 
[5] S. Fidanza, R. Minasian and A. Tomasiello, "Mirror symmetric SU(3)-structure manifolds with NS fluxes," arXiv:hep-th/0311122.

[6] C. Vafa, "Superstrings and topological strings at large N," J. Math. Phys. 42 (2001) 2798 [arXiv:hep-th/0008142].

[7] S. Gurrieri, J. Louis, A. Micu and D. Waldram, "Mirror symmetry in generalized Calabi-Yau compactifications," Nucl. Phys. B 654, 61 (2003) [arXiv:hep-th/0211102].

[8] N. Berkovits, "Super-Poincare covariant quantization of the superstring," JHEP 0004, 018 (2000) [arXiv:hep-th/0001035].

[9] H.B. Lawson and M.L. Michelsohn, "Spin Geometry," Princeton Univ. Press, 1989.

[10] S. Hellerman, J. McGreevy and B. Williams, "Geometric constructions of nongeometric string theories," JHEP 0401 (2004) 024 [arXiv:hep-th/0208174].

[11] A. Flournoy, B. Wecht and B. Williams, "Constructing nongeometric vacua in string theory," arXiv:hep-th/0404217.

[12] U. Lindstrom, "Generalized $\mathrm{N}=(2,2)$ supersymmetric non-linear sigma models," arXiv:hep-th/0401100.

[13] A. S. Cattaneo and G. Felder, "A path integral approach to the Kontsevich quantization formula," Commun. Math. Phys. 212 (2000) 591 [arXiv:math.qa/9902090].

[14] S. J. Gates, C. M. Hull and M. Rocek, "Twisted Multiplets And New Supersymmetric Nonlinear Sigma Models," Nucl. Phys. B 248 (1984) 157.

[15] S. Lyakhovich and M. Zabzine, "Poisson geometry of sigma models with extended supersymmetry," Phys. Lett. B 548 (2002) 243 [arXiv:hep-th/0210043].

[16] S. F. Hassan, "O(D,D:R) Deformations Of Complex Structures And Extended World Sheet Supersymmetry," Nucl. Phys. B 454 (1995) 86 [arXiv:hep-th/9408060].

[17] U. Lindstrom and M. Zabzine, " $\mathrm{N}=2$ boundary conditions for non-linear sigma models and Landau-Ginzburg models," JHEP 0302 (2003) 006 [arXiv:hep-th/0209098].

[18] U. Lindstrom and M. Zabzine, "D-branes in $\mathrm{N}=2$ WZW models," Phys. Lett. B 560 (2003) 108 [arXiv:hep-th/0212042].

[19] A. Kapustin, "Topological strings on noncommutative manifolds," arXiv:hepth/0310057. 
[20] P. Grange, "Branes as stable holomorphic line bundles on the non-commutative torus," arXiv:hep-th/0403126.

[21] L. Baulieu, A. S. Losev and N. A. Nekrasov, "Target space symmetries in topological theories. I," JHEP 0202 (2002) 021 [arXiv:hep-th/0106042].

[22] N. Seiberg and E. Witten, "String theory and noncommutative geometry," JHEP 9909 (1999) 032 [arXiv:hep-th/9908142].

[23] N. Ikeda, "Two-dimensional gravity and nonlinear gauge theory," Annals Phys. 235 (1994) 435 [arXiv:hep-th/9312059].

[24] P. Schaller and T. Strobl, "Poisson structure induced (topological) field theories," Mod. Phys. Lett. A 9 (1994) 3129 [arXiv:hep-th/9405110].

[25] C. Klimcik and T. Strobl, "WZW-Poisson manifolds," J. Geom. Phys. 43 (2002) 341 [arXiv:math.sg/0104189].

[26] T. Courant, "Dirac manifolds," Trans. Amer. Math. Soc. 319 (1990), no. 2, 631-661.

[27] T. Courant and A. Weinstein, "Beyond Poisson structures," Action hamiltoniennes de groupes. Troisième théorème de Lie (Lyon, 1986), 39-49, Travaux en Cours, 27, Hermann, Paris, 1988.

[28] G. Cavalcanti and M. Gualtieri, "Generalized complex structures on nilmanifolds," arXiv:math.DG/0404451. 